\title{
Response of pulmonary tuberculomas to anti-tuberculous treatment
}

\author{
H.S. Lee, J.Y. Oh, J.H. Lee, C.G. Yoo, C-T. Lee, Y.W. Kim, S.K. Han, Y-S. Shim, J-J. Yim
}

Response of pulmonary tuberculomas to anti-tuberculous treatment. H.S. Lee, J.Y. Oh, J.H. Lee, C.G. Yoo, C-T. Lee, Y.W. Kim, S.K. Han, Y-S. Shim, J-J. Yim. (C)ERS Journals Ltd 2004.

ABSTRACT: Pulmonary tuberculomas are well-circumscribed masses caused by Mycobacterium tuberculosis. However, the response of tuberculomas to antituberculous (TB) treatment has not been well defined as yet.

The response of pulmonary tuberculomas to anti-TB treatment was retrospectively reviewed in 45 patients diagnosed between January 1997 and December 2001. The areas of pulmonary tuberculomas were estimated by calculating products of the longest and their perpendicular short diameters on chest radiographs. The response to anti-TB treatment was categorised as "decreased" ( $>25 \%$ reduction in area versus its initial area), "increased" ( $>25 \%$ increase) and "no change" (the remainder).

The mean of treatment duration was $11.5 \pm 3.6$ months. Three months after treatment, 18 patients $(40.0 \%)$ were categorised as decreased, $25(55.6 \%)$ as no change and two $(4.4 \%)$ as increased. Twelve months after treatment, out of 42 patients available for chest radiographs, 32 patients $(\mathbf{7 6 . 2} \%)$ were categorised as decreased, nine $(21.4 \%)$ as no change and one patient $(2.4 \%)$ as increased. At the last follow-up (mean follow-up $\mathbf{2 7 . 0} \pm \mathbf{1 0 . 2}$ months), 37 patients $(\mathbf{8 2 . 2} \%)$ were categorised as decreased.

The majority of pulmonary tuberculomas were decreased by anti-tuberculosis treatment during and even after treatment, although a transient enlargement during the early period of treatment was observed infrequently.

Eur Respir J 2004; 23: 452-455.
Division of Respiratory and Critical Care Medicine, Dept of Internal Medicine and Lung Institute of Medical Research Centre, Seoul National University College of Medicine, Seoul, Republic of Korea.

Correspondence: J-J. Yim, Division of Respiratory and Critical Care Medicine, Dept of Internal Medicine and Lung Institute of Medical Research Center, Seoul National University College of Medicine, 28 YongonDong, Chongno-Gu, Seoul, 110-744, Republic of Korea.

Fax: 8227629662

E-mail: yimjj@snu.ac.kr

Keywords: Anti-tuberculous treatment, pulmonary tuberculoma, pulmonary tuberculosis

Received: July 282003

Accepted after revision: November 252003

This study was supported by a grant from the Korea Health 21 R\&D Project, Ministry of Health \& Welfare, Republic of Korea (00-PJ1PG1-CH03-0001).
A pulmonary tuberculoma is a well-circumscribed nodule or a mass located in the lungs and caused by Mycobacterium tuberculosis [1]. Pathologically, it is a caseous mass encapsulated by multiple concentric layers of connective tissue without surrounding inflammation or spread [2, 3]. Although pulmonary tuberculosis (TB) represents $80-90 \%$ of all TB infections in patients without human immunodeficiency virus infection, a pulmonary tuberculoma is an uncommon manifestation of pulmonary TB [4]. Pulmonary tuberculomas can be the manifestation of both primary and post-primary TB and were reported to be manifested in 6-9\% of adult-onset or post-primary TB [5-7]. The proposed strong mechanism underlying the development of tuberculomas consists of repeated extension of bronchopneumonic foci, subsequent necrosis and re-encapsulation of its capsule [3, 8]. Shrinkage of occluded cavities and fusion of small encapsulated densities were suggested as other mechanisms [1, 2, 8, 9].

The natural courses of pulmonary tuberculomas are known from reports issued before the development of effective antiTB chemotherapy. In these reports, the natural courses of tuberculomas without anti-TB chemotherapy were classified into progressive, stationary and regressive, and stationary course accounted for $30-50 \%$ of all patients [9-12]. Few reports have been issued about the response of tuberculomas to the introduction of effective anti-TB drugs. AUERBACH et al. [13] observed a greater reduction in the perifocal reaction surrounding tuberculous foci in 1948 and a greater thinning of the capsules surrounding necrotic foci in 1955 upon administering anti-TB treatment, respectively [13, 14].
However, their observations were confined to microscopical changes without mention of gross changes on chest radiographs. Moreover, their observations were made long before the introduction of the short-course, multi-drug, anti-TB treatment. In this context, the current authors evaluated the response of tuberculomas to anti-TB treatment in terms of changes in size of tuberculomas on follow-up chest radiographs.

\section{Methods and materials}

\section{Study subjects}

The medical records of patients with pulmonary tuberculoma, diagnosed bacteriologically or pathologically by percutaneous needle aspiration or biopsy, from January 1997 to December 2001 in Seoul National University Hospital (Seoul, Republic of Korea), were reviewed. Inclusion criteria for the tuberculoma were as follows: 1) the presence of acid fast bacilli (AFB) or chronic granulomatous inflammation with caseation necrosis on the pathological specimen obtained by percutaneous needle aspiration or biopsy; or 2) in case of chronic granulomatous inflammation without caseation necrosis on the pathological specimen, a positive result for mycobacterial culture from sputum or a bronchial washing fluid. Patients who did not complete their anti-TB treatment, lacked available follow-up chest radiographs, underwent surgical resection of the tuberculoma or were diagnosed as 
opportunist mycobacterial diseases were excluded. In all patients, the following were reviewed: symptoms, signs and past medical history; regimens and duration of anti-TB treatment; and radiographical features of tuberculomas.

\section{Estimation of size change}

The medical records and the chest radiographs of study subjects were reviewed. Response of tuberculomas to anti-TB treatment was evaluated by determining changes in size. For this purpose, the areas of tuberculomas were estimated by calculating products of the longest and their perpendicular short diameters on chest radiographs. In case of the patients with two or more tuberculomas, the size of the tuberculoma targeted for aspiration or biopsy was measured. The longest and their perpendicular short diameters of tuberculomas were measured on each chest radiograph obtained before treatment, during the 3rd, 6th, 9th and 12th month of treatment, and at the time of the last follow-up. For more accurate measurement, two pulmonologists respectively made three measurements and the average values were used for analysis. The relative ratios of the areas of tuberculomas were then calculated after each treatment with respect to the pretreatment areas. Using these relative area ratios, the subjects were classified into each response group: "decreased" ( $>25 \%$ reduction); "increased" (>25\% increase); and "no change" (the remainder).

\section{Statistical analysis}

Mean \pm SD of the relative area ratios was calculated at each time point and these data were analysed using the paired t-test. In addition, the t-test and the Chi-squared test were used for comparison of treatment response between different groups. A p-value of $<0.05$ was considered statistically significant.

\section{Results}

\section{Characteristics of the patients}

Forty-five patients with tuberculoma were included in the study. The male-to-female ratio was $32: 13$ and their median age was 53 yrs (range $27-71$ yrs). Seven patients (15.6\%) had a history of previous TB and 13 had diabetes mellitus $(28.9 \%)$. Twenty-one $(46.7 \%)$ out of 45 patients were symptomatic. The most frequent symptoms were sputum, cough, general weakness and fatigue. The other 24 patients visited the clinic for the evaluation of a lung mass found during routine checkup. Twenty-one $(46.7 \%)$ out of 45 patients were current smokers and four $(8.9 \%)$ were ex-smokers. The median cumulative duration of smoking was 25 pack-yrs (2-60). Pulmonary function tests were performed on 36 patients. Of these patients, five showed obstructive pattern and two revealed restrictive pattern of lung function (table 1).

Out of 45 patients, $21(46.7 \%)$ were diagnosed as having a tuberculoma by the presence of AFB on percutaneous needle aspiration or biopsy specimen, $21(46.7 \%)$ by pathology of chronic granulomatous inflammation with caseation necrosis and three patients $(6.6 \%)$ having chronic granulomatous inflammation without caseation on pathology by a positive mycobacterial culture from sputum or bronchoscopic washing fluid. AFB were observed for $21(65.6 \%)$ out of 32 stained pathological specimens. The result of the sputum AFB smear was positive for four $(10.5 \%)$ out of 38 patients before
Table 1.-Baseline characteristics of the study subjects

\begin{tabular}{lc}
\hline Total patients $n$ & 45 \\
Male:female $\mathrm{n}$ & $32: 13$ \\
Age yrs & $53(27-71)$ \\
Past TB history & $7(15.6)$ \\
Presence of diabetes mellitus & $13(28.9)$ \\
Smoking & \\
Nonsmoker & $20(44.4)$ \\
Ex-smoker & $4(8.9)$ \\
Current smoker & $21(46.7)$ \\
Cumulative amount & \\
Pulmonary function test $n$ & $25(2-60)$ \\
Normal & 36 \\
Obstructive pattern & 29 \\
Restrictive pattern & 5 \\
Symptomatic patients at the time of admission & 2 \\
Sputum & $21(46.7)$ \\
Cough & $11(24.4)$ \\
General weakness/easy fatigability & $9(20.0)$ \\
Weight loss & $10(22.2)$ \\
Chest pain & $7(15.6)$ \\
Dyspnoea on exertion & $5(11.1)$ \\
Dizziness & $4(8.9)$ \\
Sweating & $3(6.7)$ \\
Headache & $2(4.4)$ \\
Fever & $2(4.4)$ \\
Haemoptysis & $1(2.2)$ \\
Arthralgia & $1(2.2)$ \\
& $1(2.2)$ \\
\hline
\end{tabular}

Data are presented as $\mathrm{n}(\%)$ or median (range) unless otherwise stated.

\#. cumulative amount of smoking of ex-smokers and current smokers.

percutaneous needle aspiration or biopsy, and the culture was positive for 19 patients $(50.0 \%)$, including four with positive AFB smear. Bronchoscopy was performed on 22 patients and no abnormalities were found in any patient. The result of the AFB smear of bronchial washing fluid was positive for two patients and the culture was positive for five patients. Thirtyfive patients $(77.8 \%)$ had one nodule and $10(22.2 \%)$ had two or more. Thirty-one nodules $(68.9 \%)$, targeted for percutaneous needle aspiration or biopsy were located in both upper lobes (table 2).

\section{Size changes of tuberculomas with treatment}

The means of initial long and short diameters were $32.1 \pm 17.8$ and $23.2 \pm 10.8 \mathrm{~mm}$, respectively. The mean duration of anti-TB treatment was $11.7 \pm 3.7$ months (6-25). Isoniazid, rifampicin, ethambutol and pyrazinamide were prescribed for 38 patients initially, and isoniazid, rifampicin

Table 2. - Baseline characteristics of pulmonary tuberculomas

\begin{tabular}{lc}
\hline Characteristics of nodules & Patients n (\%) \\
\hline No of nodules on chest CT & \\
1 & $35(77.8)$ \\
2 & $4(8.9)$ \\
3 & $4(8.9)$ \\
5 & $2(4.4)$ \\
Location of 45 major nodules & \\
Right upper lobe & $15(33.3)$ \\
Left upper lobe & $16(35.6)$ \\
Right lower lobe & $5(11.1)$ \\
Left lower lobe & $9(20.0)$ \\
Long diameter & mm \\
Short diameter & $32.1 \pm 17.8$ \\
\hline
\end{tabular}

CT: computed tomography. ${ }^{\#}$ : data are presented as mean \pm SD. 
and ethambutol were prescribed for another six patients. Ethambutol, streptomycin, cycloserine and levofloxacin were initially given for one patient with poor liver function. The mean treatment duration of the patients treated with regimens including pyrazinamide was shorter than that of the patients treated without pyrazinamide ( $11.1 \pm 3.5$ versus $14.3 \pm 4.1$ months, $\mathrm{p}=0.03)$. Six patients $(13.3 \%)$ required a regimen change to second-line drugs due to hepatotoxicity. All patients were treated and followed-up at the present authors' hospital on a monthly basis without directly observed therapy. The mean follow-up duration was $27.0 \pm 10.2$ months (12-53).

During the 3rd month after the initiation of anti-TB treatment, 18 patients $(40.0 \%)$ were in the decreased group (nodule disappeared in one patient), $25(55.6 \%)$ in the no change group and two $(4.4 \%)$ in the increased group. The proportions of the patients in the decreased group at months 6, 9 and 12 of anti-TB treatment were $57.1,59.5$ and $76.2 \%$, respectively (fig. 1). According to the last chest radiographs $27.0 \pm 10.2$ months after the initiation of treatment, 37 patients $(82.2 \%)$ were in the decreased group (complete resolution in 14 patients, $31.1 \%$ ), seven in the no change group (15.6\%) and one patient was in the increased group $(2.2 \%)$. Changes in relative area ratios versus initial area are summarised in table 3 .

The single patient classified in the increased group till the last follow-up showed an increase in tuberculoma size versus baseline at months 3, 6, 9 and 12 and at the last follow-up. However, the size of his tuberculoma was largest during the 3rd month and started to decrease from then onwards, although it remained larger than the initial tumour.

In eight out of 10 patients with multiple nodules on chest computed tomography, the other nodules, excluding the main

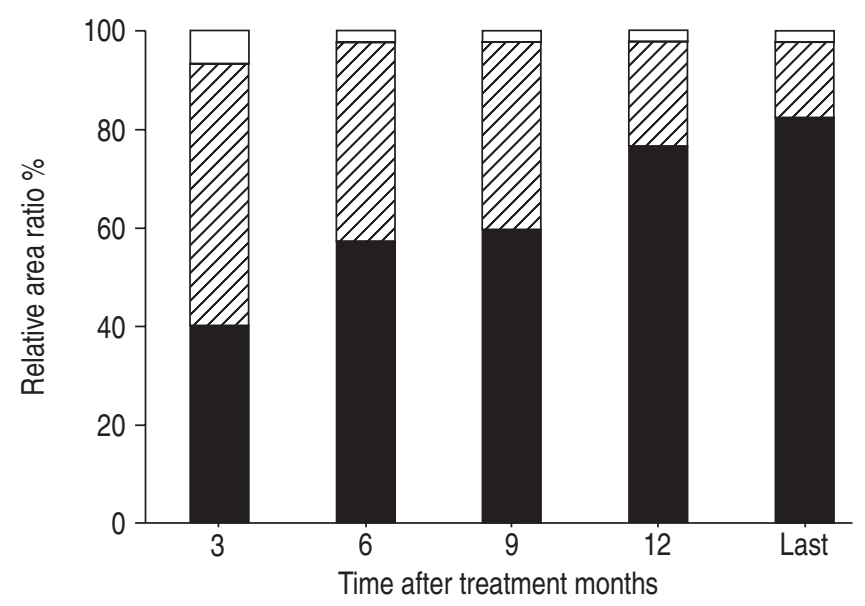

Fig. 1.-Change of the proportions of each response group with antituberculosis treatment. $\square$ : increased; $\mathbb{Z}$ : no change; $\mathbf{\square}$ : decreased.

Table 3.-Change in relative ratio of the estimate area of tuberculoma after initiation of treatment versus its initial area

\begin{tabular}{lccc}
\hline $\begin{array}{l}\text { Time after initiation } \\
\text { of treatment }\end{array}$ & $\begin{array}{c}\text { Subjects } \\
\mathrm{n}\end{array}$ & $\begin{array}{c}\text { Relative } \\
\text { ratio }\end{array}$ & p-value $^{\text {- }}$ \\
\hline 3 months & 45 & $89.9 \pm 101.0$ & 0.507 \\
6 months & 42 & $71.4 \pm 57.2$ & 0.014 \\
9 months & 42 & $62.1 \pm 49.8$ & 0.001 \\
12 months & 42 & $55.4 \pm 44.1$ & $<0.001$ \\
Last follow-up & 45 & $40.7 \pm 44.9$ & $<0.001$ \\
\hline
\end{tabular}

Relative ratio of initial area $=100$. Relative ratios are presented as mean \pm SD. \#: number of patients whose chest radiographs are available at specific time point; ": comparison between intervals. nodule targeted for aspiration or biopsy, were evaluated serially on simple chest radiographs. The other nodules of seven patients decreased with treatment, in the same way as the main nodules. However, the other nodule of one patient classified as increased, decreased in size with treatment.

No association was observed with multivariate analysis between the treatment responses and the clinical characteristics, including the presence of diabetes mellitus, a history of previous tuberculosis, inclusion of pyrazinamide in the initial regimen and duration of the treatment.

\section{Discussion}

A pulmonary tuberculoma is one of the most common benign nodules and represents $5-24 \%$ of resected solitary pulmonary nodules [15-17]. The size of pulmonary tuberculomas varies from $<1 \mathrm{~cm}$ to $>10 \mathrm{~cm}$ in diameter $[1,8]$. Tuberculomas are usually found as a single nodule, although multiple nodules are not uncommon [10]. They may include a cavity or a calcification $[1,8,18-20]$, and their margins are usually smooth and sharp [17, 19-21]. Tuberculomas are usually found in the upper lobes [1, 6, 15, 22, 23].

The diagnosis of a pulmonary tuberculoma remains a challenge, not only because it usually requires an invasive procedure, such as percutaneous needle aspiration or open thoracotomy, but also because it may be accompanied by malignancy in rare cases $[15,24,25]$. In this context, it is essential for the clinician to be aware of the treatment response of tuberculomas. In the current study, tuberculomas decreased in size at month 3 after the initiation of treatment in $40 \%$ of patients, but their size increased in two patients $(4.4 \%)$. Subsequently, however, more patients were classified as having decreased tuberculomas, even after the cessation of medication. At the time of the last follow-up, 37 patients $(82.2 \%)$ were classified in the decreased group. Given that tuberculoma size was found to decrease in only one third of cases [11] or to enlarge in $21.7 \%$ of cases [12], without effective chemotherapy, the current anti-TB treatment is probably effective on pulmonary tuberculomas in terms of size reduction. In addition, the authors did not experience bronchogenic spreading after cavitation of a tuberculoma, which was frequently observed before the introduction of chemotherapy [10-12]. The reason why the size of a tuberculoma decreases after the completion of treatment is unclear. It might be due to an accelerated natural course of healing after the eradication of $M$. tuberculosis by the anti-TB treatment.

Although increments in tuberculoma size after treatment can be explained by a paradoxical worsening observed during the early treatment period of pulmonary TB or TB lymphadenitis [26-29], the presence of an increasing tuberculoma places the clinician in a dilemma. As cases of a single pulmonary nodule containing both tuberculosis and malignancy have been reported $[15,24,25]$ and the misdiagnosis of malignancy is always possible [24], the clinician cannot be reassured of making a decision if the size of a tuberculoma increases with treatment. Surgical resection may offer an answer in such cases [19]. However, the fact that the repeated biopsy for the increasing tuberculomas of two patients yielded the same result and that these tuberculomas eventually decreased in size with treatment provides a reason for waiting whilst placing such patients under close observation.

The authors of this study regarded a mass with granuloma and caseation necrosis as a tuberculoma. Although such pathology of granuloma with caseation necrosis can be observed in fungal infections, such as histoplasmosis, blastomycosis and coccidioidosis, only imported cases that 
developed after travel to endemic areas have been reported in Korea. In terms of treatment duration, the mean treatment duration was 11.7 months, much longer than the standard recommended duration of 6 months. The lack of precise data on the evolution of tuberculomas with anti-TB treatment might make the clinician prolong the treatment duration.

In conclusion, the size of pulmonary tuberculomas was usually reduced by anti-TB treatment, although a transient enlargement during the early period of treatment had been observed infrequently.

\section{Conclusions}

The majority of pulmonary tuberculomas were reduced in size by anti-tuberculosis treatment and this tendency continued after the cessation of anti-tuberculosis treatment. However, during the early period of treatment, a tuberculoma may increase in size and thus, in such cases, decisions to place the patient under observation or to re-evaluate using invasive methods should be considered carefully.

\section{References}

1. Sochocky S. Tuberculoma of the lung. Am Rev Tuberc 1958; 78: 403-410.

2. Culver GJ, Concannon JP, McManus JE. Pulmonary tuberculomas: pathogenesis, diagnosis, and management. J Thorac Surg 1950; 20: 798-822.

3. Pratt PC. Pathology of tuberculosis. Semin Roentgenol 1979; 14: 196-203.

4. Rom WN, Garay SM. Tuberculosis. New York, Little, Brown and Company, 1996; pp. 373-412.

5. Khan MA, Kovnat DM, Bachus B, Whitcomb ME, Brody JS, Snider GL. Clinical and roentgenographic spectrum of pulmonary tuberculosis in the adult. Am J Med 1977; 62: 31-38.

6. Krysl J, Korzeniewska-Kosela M, Muller NL, FitzGerald JM. Radiologic features of pulmonary tuberculosis: an assessment of 188 cases. Can Assoc Radiol J 1994; 45: 101107.

7. Woodring $\mathrm{JH}$, Vandiviere HM, Fried AM, Dillon ML, Williams TD, Melvin IG. Update: the radiographic features of pulmonary tuberculosis. AJR Am J Roentgenol 1986; 146 : 497-506.

8. Palmer PE. Pulmonary tuberculosis - usual and unusual radiographic presentations. Semin Roentgenol 1979; 14: 204 243.

9. Mahon HW, Forsee JH. The surgical treatment of round tuberculous pulmonary lesions (tuberculomas). $J$ Thorac Surg 1950; 19: 724-740.

10. Bleyer JM, Marks JH. Tuberculomas and hamartomas of the lung: comparative study of 66 proved cases. AJR Am J Roentgenol 1957; 77: 1013-1022.
11. Bobrowitz ID. The round pulmonary tuberculous focus. Am Rev Tuberc 1943; 47: 472-483.

12. Grenville-Mathers R. The natural history of so-called tuberculomas. J Thorac Surg 1952; 23: 251-252.

13. Auerbach O, Stemmermann GN. Anatomic changes in tuberculosis following streptomycin therapy. Am Rev Tuberc 1948; 58: 449-462.

14. Auerbach O. Pathology of tuberculosis as affected by antibiotics. Am J Surg 1955; 89: 627-636.

15. Steele JD. The solitary pulmonary nodule. $J$ Thorac Cardiovasc Surg 1963; 46: 21-39.

16. Toomes H, Delphendahl A, Manke HG, Vogt-Moykopf I. The coin lesion of the lung. A review of 955 resected coin lesions. Cancer 1983; 51: 534-537.

17. Zwirewich CV, Vedal S, Miller RR, Muller NL. Solitary pulmonary nodule: high-resolution $\mathrm{CT}$ and radiologicpathologic correlation. Radiology 1991; 179: 469-476.

18. Hillerdal O. Tuberculoma of the lung. Acta Tuberc Scand 1954; 34: 1-191.

19. Ishida T, Yokoyama H, Kaneko S, Sugio K, Sugimachi K, Hara N. Pulmonary tuberculoma and indications for surgery: radiographic and clinicopathological analysis. Respir Med 1992; 86: 431-436.

20. Lee KS, Im JG. CT in adults with tuberculosis of the chest: characteristic findings and role in management. AJR $\mathrm{Am}$ J Roentgenol 1995; 164: 1361-1367.

21. Lee KS, Song KS, Lim TH, Kim PN, Kim IY, Lee BH. Adult-onset pulmonary tuberculosis: findings on chest radiographs and CT scans. AJR Am J Roentgenol 1993; 160: 753-758.

22. Goodwin RA, Des Prez RM. Apical localization of pulmonary tuberculosis, chronic pulmonary histoplasmosis, and progressive massive fibrosis of the lung. Chest 1983; 83: 801-805.

23. Snider GL. Pulmonary tuberculosis and centrilobular emphysema: the "upright theory" of apical localization. Arch Intern Med 1963; 111: 762-771.

24. Kim YI, Goo JM, Kim HY, Song JW, Im JG. Coexisting bronchogenic carcinoma and pulmonary tuberculosis in the same lobe: radiologic findings and clinical significance. Korean J Radiol 2001; 2: 138-144.

25. Ting YM, Church WR, Ravikrishnan KP. Lung carcinoma superimposed on pulmonary tuberculosis. Radiology 1976; 119: 307-312.

26. Campbell IA, Dyson AJ. Lymph node tuberculosis: a comparison of various methods of treatment. Tubercle 1977; 58: 171-179.

27. Leung AN, Muller NL, Pineda PR, FitzGerald JM. Primary tuberculosis in childhood: radiographic manifestations. Radiology 1992; 182: 87-91.

28. Steer A. A study of healing and repair of pulmonary tuberculous lesions with and without chemotherapy. Am Rev Respir Dis 1967; 95: 209-219.

29. Weber AL, Bird KT, Janower ML. Primary tuberculosis in childhood with particular emphasis on changes affecting the tracheobronchial tree. Am J Roentgenol Radium Ther Nucl Med 1968; 103: 123-132. 